\title{
Integrated nucleosynthesis in neutrino-driven winds
}

\author{
L. Huther ${ }^{1 *}$, T. Fischer ${ }^{1}$, G. Martínez-Pindeo ${ }^{1,2}$ \& K. Langanke ${ }^{2,3}$ \\ ${ }^{1}$ TU Darmstadt, \\ ${ }^{2}$ GSI Helmholtzzentrum für Schwerionenforschung, \\ ${ }^{3}$ Frankfurt Institute of Advanced Studies
}

\begin{abstract}
We analyze the explosive nucleosynthesis in neutrino-driven winds using state-of-the-art 1dimensional general relativistic radiation hydrodynamics core-collapse supernova simulations including spectral three flavor Boltzmann neutrino transport. These studies have been done for three simulations with different progenitor masses and carried out for times including the complete deleptonization of the proto-neutron star. Our results are compared with yield observations of metal-poor stars.
\end{abstract}

XII International Symposium on Nuclei in the Cosmos,

August 5-12, 2012

Cairns, Australia

${ }^{*}$ Speaker. 


\section{Introduction}

Massive stars end their lives in core collapse supernova explosions. Although the detailed explosion mechanism is still subject to research, it is expected that a large fraction of these explosions will produce a neutron star that during the first few tens of seconds will cool by emission of neutrinos of all flavor. A part of these neutrinos will transfer energy to material on top of the proto-neutron star and producing an outflow of material known as neutrino-driven wind (NDW). NDW have been considered for a long time as a possible site for r-process nucleosynthesis [1].

Recent advances in core-collapse supernova modeling allow now for spherically symmetric long term Boltzmann neutrino transport simulations covering the whole period of deleptonization of the proto-neutron star $[2,3]$. These simulations show that the matter ejected in the neutrinodriven wind is predominantly proton-rich with $Y_{e}>0.5$ and typical entropies of 50-100 $k_{b} /$ baryon. These conditions are not favorable for the r-process [4]. However the proton richness of the ejecta together with the high neutrino fluxes of the cooling proto-neutron star favors $v$ p-process nucleosynthesis [5]. In this work we want to study if the vp-process can account for the observed abundance pattern from metal poor stars [6]. Previous work has been based on fully parametric wind models [7] or in hydrodynamical simulations that treated the $Y_{e}$ of the ejecta and its evolution as a free parameter [8]. We base our study on state of the art simulations that consistently describe neutrino-matter interactions and furthermore we would like to focus our attention on the dependence of the nucleosynthetic results with progenitor mass.

\section{Nucleosynthesis}

We have performed NDW nucleosynthesis studies based on radiation hydrodynamics simulations of ref. [2]. Our calculations are based on an electron-capture supernova of mass $8.8 \mathrm{M}_{\odot}$ [9] and two iron core progenitors of masses $10.8 \mathrm{M}_{\odot}$ and $18.0 \mathrm{M}_{\odot}$ [10]. The possibility that elements beyond iron up to $\mathrm{Z}=50$ can be produced in electron-capture supernova have recently been explored in ref. [11].

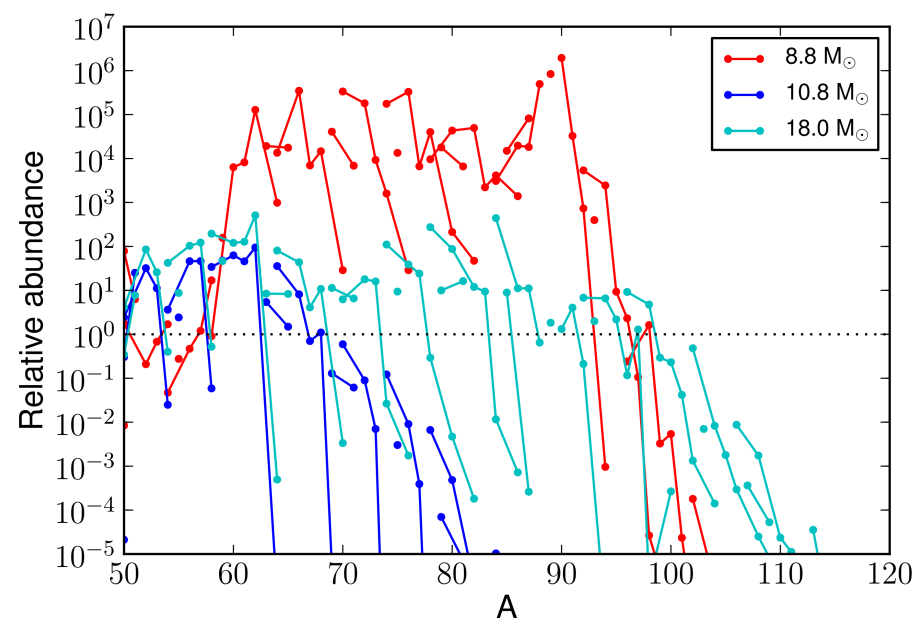

Figure 1: Relative isotopic abundance pattern of the three used models with different progenitor masses. For further details see the text below. 

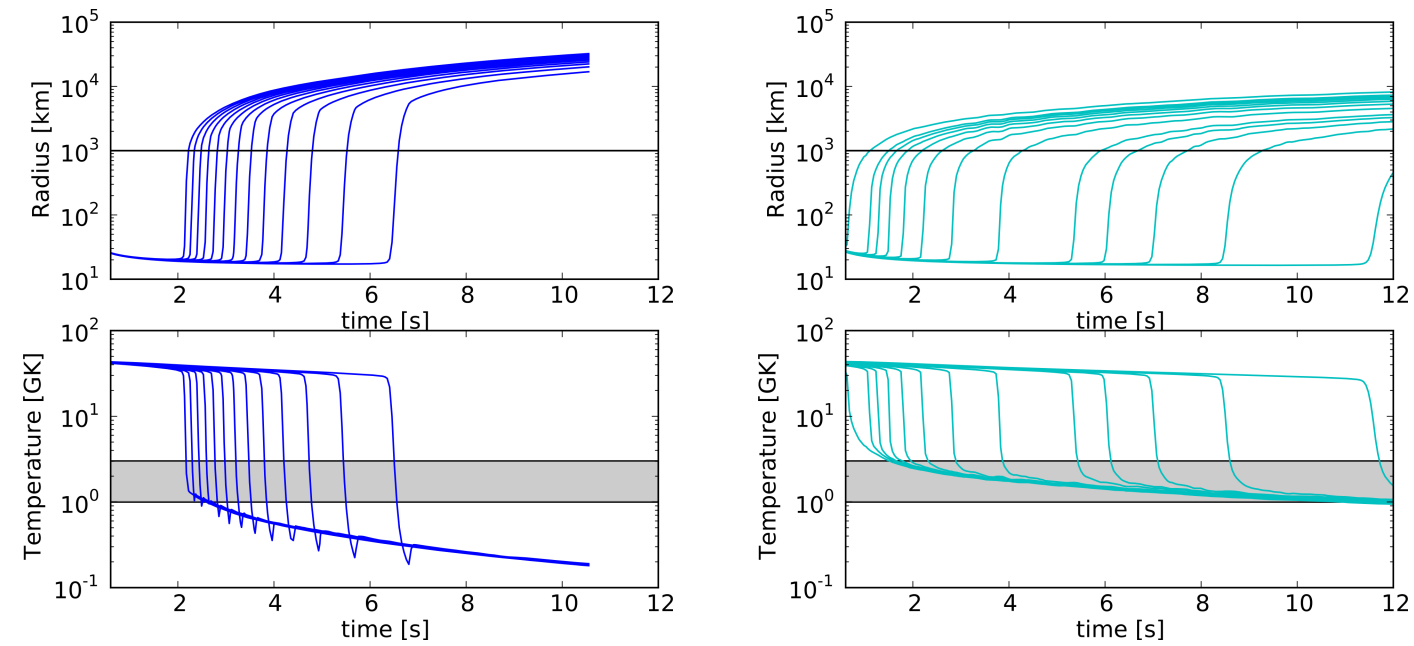

Figure 2: Temperature and radial evolution of different trajectories of the NDW. The left hand side shows the evolution of the $10.8 \mathrm{M}_{\odot}$ progenitor simulation whereas the right side represents trajectories for the $18.0 \mathrm{M}_{\odot}$ model. The marked area in the temperature plot represents the temperature range in which the $v$ p-process works efficient. For temperatures higher than 3-4 GK quasi statistical-equilibrium is dominating, whereas for temperatures lower than $1 \mathrm{GK}$ proton captures are slowed down due to Coulomb repulsion [7].

We performed the nucleosynthesis studies using a large nuclear network, including all the relevant rates, on around 100 stellar trajectories for each supernova simulation. If the nucleosynthesis outcome was not fixed at the end of the supernova simulations, we extended the simulations following an adiabatic expansion similar to that given in [7].

To obtain the integrated abundances of each simulation, we weighted the nucleosynthesis results of each trajectory with its corresponding mass content. Thus the relative abundance of element $i$ is defined as

$$
P_{i}=\sum_{m} \frac{X_{i, m} M_{m}}{X_{i, \odot} M_{\text {wind }}}
$$

where $M_{\text {wind }}=\sum_{m} M_{m}$ is the total mass ejected in the wind.

In figure 1 we show the obtained relative abundances of the three models studied here. One can observe that for the $10.8 \mathrm{M}_{\odot}$ model only elements up to $A=64$ are produced, while in the $18.0 \mathrm{M}_{\odot}$ model elements up to $A=100$ are created. To understand this we would like to refer to figure 2 that shows the evolution of temperature and density for a few selected trajectories. Due to the steep density gradient in the $10.8 \mathrm{M}_{\odot}$ model the phase of homologous expansion only stops when the wind reaches radii of $\sim 10000 \mathrm{~km}$ corresponding to temperatures below $1 \mathrm{GK}$. Such a fast expansion does not allow for an efficient $v$ p-process that is responsible for the production of elements with $A>64$ in proton-rich ejecta. In the $18.0 \mathrm{M}_{\odot}$ model the density profile is more shallow and the wind decelerates at distances around $1000 \mathrm{~km}$, corresponding to temperatures in the range 1-2 GK. This are in fact optimal conditions for an efficient $v$ p-process $[7,12]$.

The situation is different for the $8.8 \mathrm{M}_{\odot}$ progenitor. In this case much larger relative abundances in the range $A=60-100$ are obtained. They are mainly produced in slightly neutron rich material with $Y_{e}=0.46$ that are dynamically ejected by the supernova shock before the later protonrich NDW. 


\section{Comparison with metal-poor star observation}

In this section we would like to present a comparison of the elemental abundance obtained for various progenitor models with observations of the metal-poor star HD 122563 [6]. The elemental abundance is defined as $Y(Z)=\sum_{m, A} Y_{m}(A, Z) M_{m} / M_{e j}$ with the sum including those regions where iron group and heavier elements are produced and $M_{e j}$ the total mass contained in those regions. The left panel of figure 3 shows the elemental abundances of the 10.8 and $18.0 \mathrm{M}_{\odot}$ star compared with the elemental abundance pattern observed in HD 122563 that has been arbitrarily normalized to the computed iron abundance of the $18.0 \mathrm{M}_{\odot}$ model. While it is clear that the $10.8 \mathrm{M}_{\odot}$ model can not account for the observations in the range $Z=38-50$, the situation is better for the $18.0 \mathrm{M}_{\odot}$ model. In our studies we could observe that by reducing the expansion velocity of the wind, we could improve the efficiency of the $v$ p-process enhancing the abundances of elements with $Z>38$ and bringing it in agreement with observations. This slower wind ejecta may be expected in explosions of more massive progenitors. In the right panel of figure 3 the elemental abundances of the $8.8 \mathrm{M}_{\odot}$ progenitor model are compared to HD 122563. Due to the fact that in electron capture supernovae it is not expected that a substantial amount of iron is produced, we therefore normalized the observations to the computed $Z=40$ abundance. As stated above most of this material is produced in the slightly neutron rich ejecta shown in the figure with triangles. Ref. [11] shows that electron fraction values as low as 0.3 are required to produce elements up to $Z=50$.
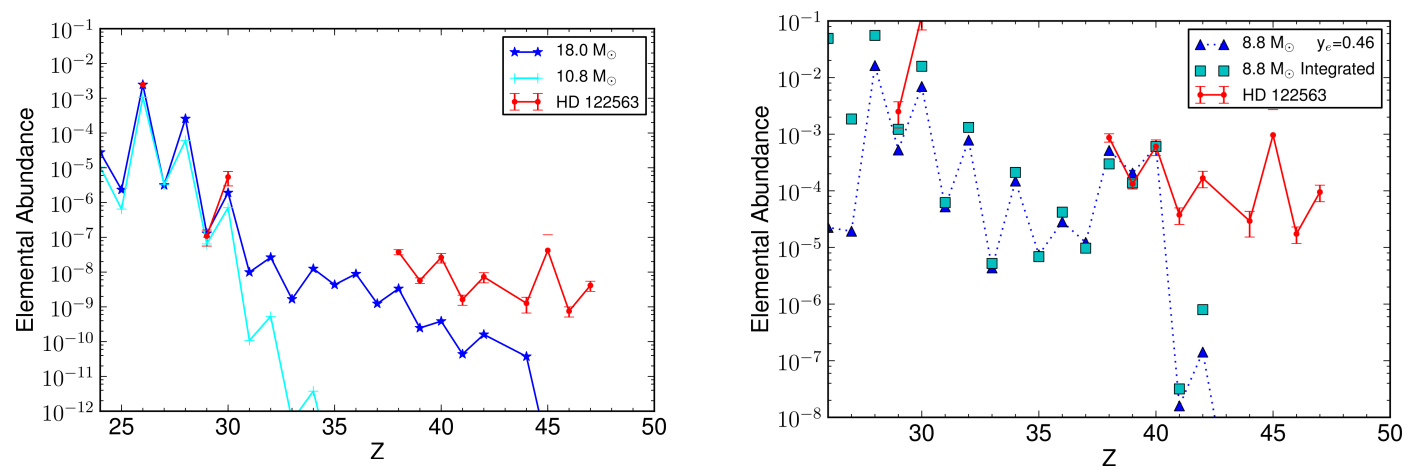

Figure 3: Elemental abundances for the $8.8,10.8$ and $18.0 \mathrm{M}_{\odot}$ models.

\section{Conclusions \& Outlook}

In this work we studied the nucleosynthetic outcome for the neutrino-driven wind of corecollapse supernovae simulations. These simulations have been based on 1-dimensional generalrelativistic radiation hydrodynamics including Boltzmann neutrino transport, covering the whole period of deleptonization. We could observe that iron core progenitors with masses around $11 \mathrm{M}_{\odot}$ cannot produce elements above iron via the $v$ p-process, since matter moves very fast to higher radii which reduces the neutrino flux. Furthermore the temperature drops very quickly below $1 \mathrm{GK}$ which also stops the $v$ p-process. Our results of current supernova simulations on heavier progenitor masses show that these stars may contribute to the abundances between $38<\mathrm{Z}<45$.

The integrated nucleosynthetic result of an electron-capture supernova with progenitor mass of $8.8 \mathrm{M}_{\odot}$ has also been analyzed. Here the elemental abundances of $\mathrm{Sr}, \mathrm{Y}$ and $\mathrm{Zr}$ could be reproduced. 
The production of heavier elements requires neutron rich ejecta with $Y_{e}$ much lower than the values considered here [11].

We would like to point out that an improved treatment of neutrino charged-current reactions at high densities has shown that the ejecta can - in contrast to ref. [2,3] - become neutron rich during deleptonisation [13]. The exact value of the electron faction is depending on properties on the equation of state as the symmetry energy. We will update our studies, once long-term simulations with improved charged-current neutrino reactions become available.

\section{Acknowledgments}

L. Huther is supported by the Deutsche Forschungsgemeinschaft through contract SFB 634. T. Fischer is supported by the Swiss National Science Foundation under project no. PBBSP2-133378. G. Martínez-Pinedo is partly supported by the Deutsche Forschungsgemeinschaft through contract SFB 634, the Helmholtz International Center for FAIR within the LOEWE program by the state Hesse.

\section{References}

[1] S. E. Woosley, J. Wilson, G. J. Mathews, R. D. Hoffman, and B. S. Meyer, The Astrophysical Journal 433, 229 (1994).

[2] T. Fischer, S. C. Whitehouse, A. Mezzacappa, F. Thielemann, and M. Liebend, Astronomy \& Astrophysics 517 (2010), arXiv: $0908.1871 v 5$.

[3] L. Hüdepohl, B. Müller, H.-T. Janka, A. Marek, and G. Raffelt, Physical Review Letters 104, 1 (2010), ISSN 0031-9007.

[4] Y.-Z. Qian and G. Wasserburg, Physics Reports 442, 237 (2007), ISSN 03701573.

[5] C. Fröhlich, G. Martínez-Pinedo, M. Liebendörfer, F.-K. Thielemann, E. Bravo, W. Hix, K. Langanke, and N. Zinner, Physical Review Letters 96, 1 (2006), ISSN 0031-9007.

[6] S. Honda, W. Aoki, Y. Ishimaru, S. Wanajo, and S. Ryan, The Astrophysical Journal 643, 1180 (2006).

[7] S. Wanajo, H.-T. Janka, and S. Kubono, The Astrophysical Journal 729, 46 (2011), ISSN 0004-637X.

[8] A. Arcones and F. Montes, The Astrophysical Journal 731, 5 (2011), ISSN 0004-637X.

[9] K. Nomoto, The Astrophysical Journal 322, 206 (1987).

[10] S. E. Woosley, A. Heger, and T. A. Weaver, Reviews of modern Physics 74, 1015 (2002).

[11] S. Wanajo, K. Nomoto, H.-T. Janka, F. S. Kitaura, and B. Müller, The Astrophysical Journal 695, 208 (2009), ISSN 0004-637X. 
[12] A. Arcones, C. Fröhlich, and G. Martínez-Pinedo, The Astrophysical Journal 750, 18 (2012), ISSN 0004-637X.

[13] G. Martínez-Pinedo, T. Fischer, A. Lohs, and L. Huther, pp. 1-5 (2012), arXiv:1205.2793v1. 